\title{
Mixing Fluids and Granular Materials
}

\author{
Toon Lenaerts and Philip Dutré
}

Computer Science Department, Katholieke Universiteit Leuven, Belgium

\begin{abstract}
Fluid animations in computer graphics show interactions with various kinds of objects. However, fluid flowing through a granular material such as sand is still not possible within current frameworks. In this paper, we present the simulation of fine granular materials interacting with fluids. We propose a unified Smoothed Particle Hydrodynamics framework for the simulation of both fluid and granular material. The granular volume is simulated as a continuous material sampled by particles. By incorporating previous work on porous flow in this simulation framework we are able to fully couple fluid and sand. Fluid can now percolate between sand grains and influence the physical properties of the sand volume. Our method demonstrates various new effects such as dry soil transforming into mud pools by rain or rigid sand structures being eroded by waves.
\end{abstract}

Categories and Subject Descriptors (according to ACM CCS): Computer Graphics [I.3.7]: Three-Dimensional Graphics and Realism-

\section{Introduction}

Fluid animations are quite popular in computer graphics animations. Physically-based simulation algorithms are usually preferred over manually modeling fluid behavior because fluids interacting with other objects or materials can be quite complex. In recent years fluids have been successfully coupled to rigid [CMT04] and deformable [GSLF05] objects, made out of both solid and porous materials [LAD08]. However, with one notable exception [RSKN08], the interaction between fluids and granular materials has still not been animated. Granular materials such as soil or sand can be considered as porous materials, which means that fluid can percolate into the empty space between grains, affecting the physical behavior of the resulting mixture. For example, dry soil can turn into mud when water is added, yet with the right amount of water sand castles become rigid structures, that are easily destroyed by a breaking wave. These are important phenomena that can be witnessed for example on rainy days or at the beach.

This paper presents a method to simulate the interaction of fluids and granular materials in a single framework. Work has been published on simulating granular materials and fluids separately, but fully coupling the two volumes seems difficult. One challenge is that fluid has to be able to flow through the open space in the granular volume, which directly couples the fluid and sand resolution at which the sim-

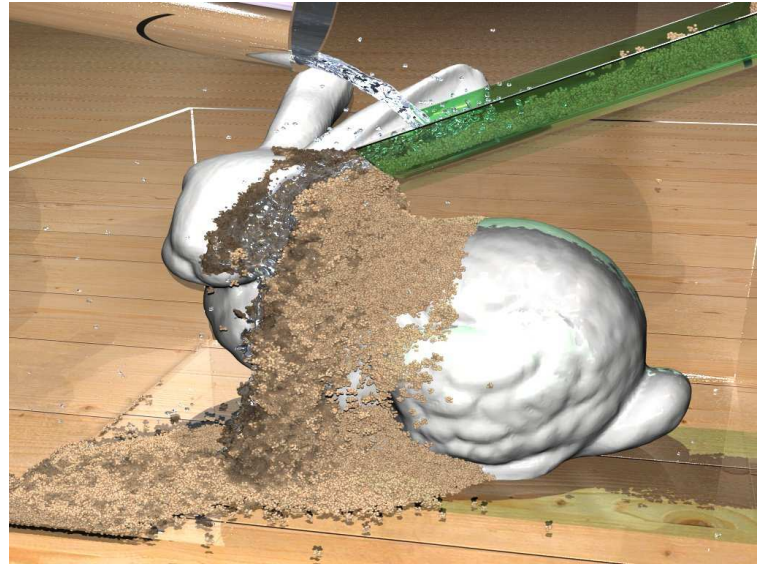

Figure 1: Dry sand slides down on a Stanford Bunny. At the same time water is poured on the sand. Moist sand piles up rigidly while wet sand turns in to viscous mud.

ulation is performed. Another difficulty is simulating of the resulting mixture, since the fluid can alter the behavior of the granular material or vica versa.

The main contribution of this work is a unified Smoothed Particle Hydrodynamics (SPH) framework where fluids and granular materials are two-way coupled. We show how sand 
can be simulated using the sand model of Zhu and Bridson [ZB05] in a particle framework, and extend the work of [LAD08] to simulate porous flow through granular materials and to model the behavior of the resulting mixture. As can be seen in Figure 1, the resulting animations show a full coupling between fluids and sand, featuring fluid percolation, erosion and mud formation.

Previous Work The animation of fluids has been researched actively in recent years. Early work on Eulerian models by Foster and Metaxas [FM96, FM97, FF01] and Stam [Sta99] showed how the incompressible three dimensional Navier-Stokes equations could be solved stably and practically on a grid. Later work two-way coupled gridbased fluids to rigid objects [CMT04], deformable bodies [CGFO06] and thin shells [GSLF05].

The Lagrangian method of solving fluid flow is another model based on particles. First introduced to the graphics community by Desbrun and Cani [DC96] to simulate highly deformable objects and later used by Müller et al. [MCG03,MSKG05] for simulating fluids at interactive rates, SPH is now a very popular method for simulating fluid flow (see Monaghan [Mon05] for a good overview). An alternative particle model is the Moving Particle Semi-Implicit (MPS) method, introduced by Premože et al. [PTB*03] to the graphics community. Like the Eulerian models, particlebased models are also able to simulate interactions between fluids and rigid objects [KAG $\left.{ }^{*} 05, \mathrm{CBP} 05, \mathrm{BTT} 09\right]$, deformable bodies [SSP07] and thin shells [LAD08]. Water absorption has been simulated on surfaces by Liu et al. [LZLW05]. The full porous flow through rigid and elastic objects was presented by Lenaerts et al. [LAD08].

Soil and sand terrains can be simulated as height-fields [LM93, CLH96]. Sumner et al. [SOH99] extended the heighfield approach to model footprints and other tracks. Also, interactive manipulations are possible [ON03, PCGFMD08]. Sand animations based on particles were introduced by Miller and Pearce [MP89]. Bell et al. [BYM05] simulated granular materials using a particle-particle collision model. Although they can handle large amounts of colliding bodies efficiently, the simulation resolution is directly linked to the grain size, which makes large or detailed sand simulations impractical within short time frames. In contrast, Zhu and Bridson [ZB05] take a continuum approach by simulating sand as a fluid, thereby decoupling the resolution of the simulation from the grain size. For a more detailed discussion of simulation models for granular materials both in computer graphics as well as in the physics community, we refer to the work of Bell et al. [BYM05] and Zhu and Bridson [ZB05].

Wojtan et al. [WCMT07] simulated sand erosion, but fluids could not percolate into the sand volume. Falappi and Gallati [FG07] coupled granular and fluid phases using SPH. Fully coupling fluids to granular materials was only recently been made possible by Rungjiratananon et al. [RSKN08]. Fluid percolation is simulated by transferring a wetness value from fluid particles to granular particles and applying a cohesion force accordingly. Although they reach interac- tive simulations by a GPU implemention, they simulate one sand grain by one particle and therefore are subject to the same scalability limitations as the model of [BYM05].

Overview A survey of our particle model is provided in section 2. After highlighting our fluid solver and porous flow framework, we discuss how the grid-particle sand simulation framework of [ZB05] can be transfered to a particle framework. Section 3 then explains how the effects of porous flow through granular materials can be incorporated in the simulation. The resulting animations are shown and discussed in sections 4 and 5. Finally, we conclude our work in section 6 .

\section{Particle Simulation Framework}

We simulate our fluids using the SPH method, which samples and represents fluid volumes as discrete particles $p_{i}$, centered at the position $\mathbf{x}_{i}$, with mass $m_{i}$, volume $V_{i}$ and material density $\rho_{i}=m_{i} / V_{i}$. Continuous properties (such as interaction forces) are derived from the discrete particles using the classical SPH equation [Mon92, Mon05]:

$$
A(\mathbf{x})=\sum_{j} V_{j} A_{j} W\left(\mathbf{x}_{j}-\mathbf{x}, h_{j}\right),
$$

where $W\left(\mathbf{x}_{j}-\mathbf{x}, h_{j}\right)$ is a radially symmetric smoothing kernel function and $h_{j}$ is the smoothing length associated with particle $p_{j}$.

Fluid Simulation Our SPH fluid framework is based on previous work [MCG03, MSKG05, BT07]. The NavierStokes momentum equation $\left(\rho \frac{D \mathbf{v}}{D t}=-\nabla P+\mu \nabla^{2} \mathbf{v}+\rho \mathbf{g}\right)$ models the behavior of fluids. Here, $\mathbf{v}$ is velocity, $P$ is pressure, $\mu$ is viscosity and $\mathbf{g}$ is the gravity. Particle forces can easily be obtained using SPH [MCG03].

Porous Flow Simulation Porous flow is simulated as in the work of [LAD08] where porous particles represent small porous volumes capable of holding an amount of fluid. Such a porous particle is characterized by the porosity $\phi$ and permeability $K$ of the porous volume, which denote the volume fraction that is void of solid material and the ability to transmit fluid respectively. Fluid percolation through the body is then simulated as a diffusion process of fluid mass between porous particles. Similarly, at the interface between the object and the fluid, fluid mass is diffused from and to fluid particles to simulate absorption and emission.

In order to apply this porous particle model to the simulation of granular materials and fluids, a simulation model that uses volumes of granular material as simulation elements, rather than simulating the individual grains, is needed. The sand-as-a-fluid model from [ZB05] fits this description. They propose a two-step simulation framework for granular materials which simulates a sand volume as a fluid on a regular grid and then applies friction forces. The resulting velocity field is used to advect particles for which a surface mesh is computed. In the following paragraph we show how this grid-based model can be transferred to a particle-based model. 
Granular Simulation In our Langrangian setting the sand volume is sampled with particles similar to fluids. Compared to previous particle-based sand models, this continuum approach enables more efficient simulations of sand in bulk. We use our fluid SPH framework to solve for pressure gradients and make the intermediate velocity field nearly incompressible using Tait's pressure equation [BT07]. Then, a simplified stress model is used to apply friction in the sand volume. Zhu and Bridson [ZB05] decomposed the sand domain in regions moving rigidly and regions of shearing flow. The frictional stress for particles in regions of shearing flow is given as:

$$
\sigma_{f}=-\mu_{f} \frac{D}{\sqrt{1 / 3|D|_{F}}}
$$

where $\mu_{f}$ is the friction coefficient. The strain rate tensor $D=\left(\nabla \mathbf{u}+\nabla \mathbf{u}^{T}\right) / 2$ is evaluated for each particle using SPH [SSP07], where $\nabla \mathbf{u}$ is the displacement gradient:

$$
\nabla \mathbf{u}=\sum_{j} V_{j}\left(\mathbf{v}_{j} \Delta t\right) \cdot \nabla W\left(x_{j}-x_{i}, h_{j}\right) .
$$

Particles in regions moving rigidly can be found by testing the rigid stress $\sigma_{r}$ :

$$
\sigma_{r}=-\frac{\rho D h^{2}}{\Delta t}
$$

against the Mohr-Coulomb condition, which determines material yielding

$$
\sqrt{3} \sigma_{s}<\mu_{f} \sigma_{m}+c
$$

where $\sigma_{s}$ is the shear stress, $\sigma_{m}$ the mean stress and $c$ is grain cohesion. We then search for clusters of particles marked as rigid. Two rigid particles belong to the same cluster when at least one path can be constructed between those particles over neighboring particles within a support range $h^{\prime} \leq h$ (see also Figure 2(a)). Smaller support ranges $h^{\prime}$ result in more rigid clusters which can break apart more easily. Forces on cluster particles are accumulated to a total force and torque and the particle cluster is then moved as a rigid body [KAD* $06, \mathrm{SSP} 07]$. The remaining particles are updated with the frictional stress by computing the force out of $\sigma_{f}$ similar to the work of Solenthaler et al. [SSP07].

\section{Simulating Porous Flow in Granular Materials}

A granular material can be considered as a porous material. The space between individual grains of a granular volume is the actual pore space. By choosing a simulation algorithm that simulates on volumes of grains instead of simulating the actual grains, we can use porous particles as volumes of grains and simulate porous flow exactly as in the work of Lenaerts et al. [LAD08]. Moreover, simulating sand as a fluid allows us to easily simulate a mixture of granular material and fluid and transition from dry dirt to a mud stream for example.

In a moist sand volume the surface tension of the fluid between the sand grains strengthens the sand volume to form

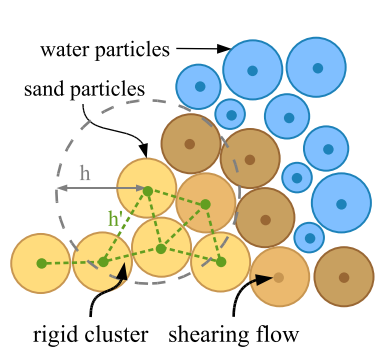

(a) Regions of sand flow

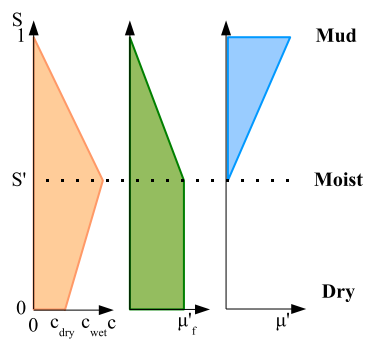

(b) Parameter evolution
Figure 2: (a) Two regions of sand flow identified by testing the Mohr-Coulomb condition: Sand particles marked as rigid (green) are clustered and moved as a whole, the other sand partciles are in a state of shearing flow and are moved seperately. (b) As fluid is absorbed, the saturation level $S$ determines sand cohesion $c$, sand friction $\mu_{f}^{\prime}$ and viscosity $\mu^{\prime}$. Dry sand transitions to a rigid moist structure to viscous mud.

a more rigid structure. At this point the sand volume has reached an ideal saturation level $S^{\prime}$. We use the saturation level to control the cohesion term $c$ in the Mohr-Coulomb condition. We linearly interpolate between a cohesion $c^{d r y}$ for the dry material and a cohesion $c^{\text {wet }}$ for the moist material, depending on the saturation level (Figure 2(b)):

$$
c=\left\{\begin{aligned}
c^{d r y}\left(1-\frac{S}{S^{\prime}}\right)+c^{\text {wet }} \frac{S}{S^{\prime}} & \text { if } S \leq S^{\prime} \\
c^{\text {wet }} \frac{1-S}{1-S^{\prime}} & \text { if } S>S^{\prime}
\end{aligned}\right.
$$

As more water is added to the mixture, sand grain spacing increases and the mixture becomes more liquid. To simulate such a mixture we add a viscosity term [MCG03] to the velocity field of the sand and use the saturation level $S$ to scale the viscosity coefficient $\mu^{\prime}=\mu\left(S-S^{\prime}\right) /\left(1-S^{\prime}\right)$ of the fluidsand mixture. At the same time, we down-scale the friction coefficient $\mu_{f}^{\prime}=\mu_{f}(1-S) /\left(1-S^{\prime}\right)$ of the sand as the saturation increases.

The net result is a sand volume that can turn in to a rigid structure or a viscous fluid volume and vice versa. The evolution of these parameters versus the saturation is plotted in Figure 2(b).

\section{Results}

The framework was implemented in $\mathrm{C}++$ and parallelized using OpenMP. All simulations ran on a 4 -core $2.66 \mathrm{GHz}$ $\mathrm{CPU}$ and 4GB of memory. The animations are rendered using POV-Ray.

Visualizing the simulation particles can result in poor sand renderings. Instead, we visualize sand volumes by rendering pseudo-random high resolution particles for each of the simulation particles. These high resolution particles are fixed per simulation particle to avoid flickering. As the sand volume saturates we gradually fade in a sand mesh to illustrate the 


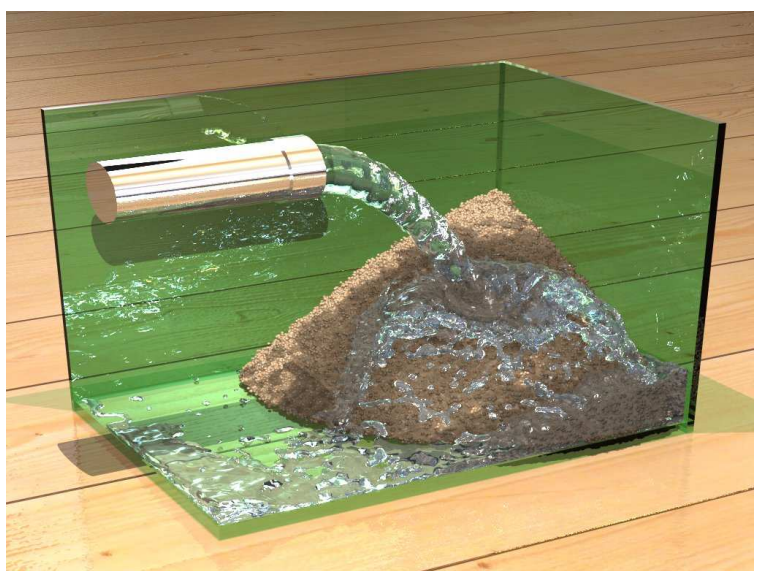

Figure 3: Water is poured on a pile of dry sand. The water percolates into the sand volume and erodes a hole in the pile.

wet look. This is achieved by using the saturation level in the alpha component of the vertex colors. Fluid and sand surfaces are extracted using the colorfield of [SSP07] and the Marching Cubes algorithm [LC87].

In Figure 3 we show a dry sand column collapsing as in [ZB05]. Once the sand pile has come to rest we pour water onto the sand. Notice how the water jet mixes with the sand and erodes a hole in the pile. The simulation consists of 45,000 sand and 35,000 water particles and took approximately $50 \mathrm{~s}$ per animation frame.

Figure 4 shows the same sand column, however now the sand is $40 \%$ saturated. The surface tension of the present water keeps the column rigidly standing, which is simulated by increasing the cohesion term as in Equation 6. A water column is released on the sand column. Notice how the water erodes the lower half of the sand column and weakens the sand structure. Eventually the sand column falls apart in the water. The container is $16 \mathrm{~cm} \times 10 \mathrm{~cm} \times 12 \mathrm{~cm}$. The simulation consists of 45,000 sand and 75,000 water particles and ran at approximately $78 \mathrm{~s}$ per frame.

A rain shower is simulated in Figure 5. At first only a few drops fall from the sky on the dry soil and can be absorbed rapidly. As more rain drops fall down, the soil becomes saturated, cannot handle the excess water and turns into a puddle of mud. The soil is simulated using 40,000 particles, the rain consisted of 50,000 particles. The average computation time was 1 minute per frame.

In Figure 1 we show dry sand sliding on the Stanford Bunny. Then, we start pouring water on the sliding sand which transforms into more rigid moist sand. Notice how moist sand piles up at the head and right next to the Bunny. In the end of the animation the excess water is also absorbed, weakening the pile and making it collapse. We use the saturation level of the sand particles to guide the stickiness [CBP05] between the surface of the Bunny and the sand. The Bunny statuette is $20 \mathrm{~cm} \times 15 \mathrm{~cm} \times 17 \mathrm{~cm}$. Approximately 70,000 particles were used in total, requiring about 1 min of computation per frame.

\section{Discussion}

The model of Zhu and Bridson [ZB05] approximates the pressure in the sand by the fluid pressure used to get an incompressible velocity field for the sand volume. In some cases (e.g. hour glass simulations) this yields inaccurate results as will our method since it is based on the same principles. Our approach uses the SPH fluid method, which cannot guarantee incompressibility, and thus inherits the same compressibility issues from SPH. However, by using Tait's equation [BT07] for the pressure computation, we obtain a weakly compressible volume $(<1 \%)$, limiting the error.

In the proposed framework, one sand particle represents a volume of sand grains. Sand particles can easily drift or splash apart when interacting with fluid. In that case, the sand particle is a poor approximation since the grains would probably spread in the fluid. Using an adaptive particle sampling approach such as in [DC99] or [APKG07] would result in a better sampling of the sand volume.

Also, the effective stress, which is the stress reduced by the absorbed fluid [LAD08], may cause fractures in the sand volume by increasing particle spacing. These fractures may be influenced by the initial particle sampling, especially for low resolution samplings. Since the porous flow algorithm requires the whole volume to be sampled with porous particles, we cannot use adaptive sampling schemes as in the framework of [PKA* 05]. The techniques of [DC99, APKG07] might be better suited.

Visualizing sand volumes is a difficult task. We employ a naive method for visualizing the simulation particles by rendering 10 smaller sand particles. This conveys the illusion of sand grains for bodies of sand and even visualizes sand flow, which would not be the case when visualizing a surface mesh. However, the illusion fails when particles drift apart. Alternative methods, such as advecting high resolution particles with the velocity field of the simulation particles or actually simulating high resolution particles at the surface, deliver more realistic results [ABC $\left.{ }^{*} 07\right]$, but are computationally quite expensive.

In concurrent work, Rungjiratananon et al. [RSKN08] presented an alternative framework for animating the interaction between sand and water. They couple an SPH fluid system to a Discrete Element Method (DEM) for the simulation of granular materials. Fluid percolation is modelled by transferring wetness values and applying a cohesion force between sand particles accordingly. Although they achieve similar effects, the DEM simulates one sand grain by one particle and therefore is subject to the same scalability limitations as the model of [BYM05]. In contrast, we simulate the sand volume as a fluid which not only decouples particle resolution from the sand grains, but also facilitates a smooth transition from dry sand to moist sand to mud. 

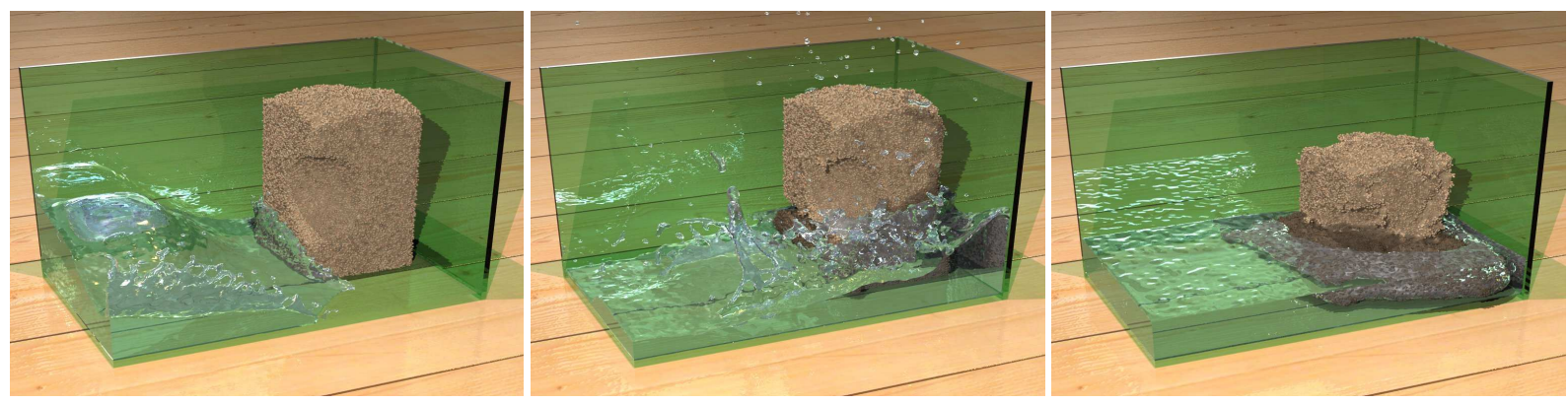

Figure 4: A moist sand column stands rigidly until a column of water is released. The water percolates through the sand and erodes pieces of the structure.
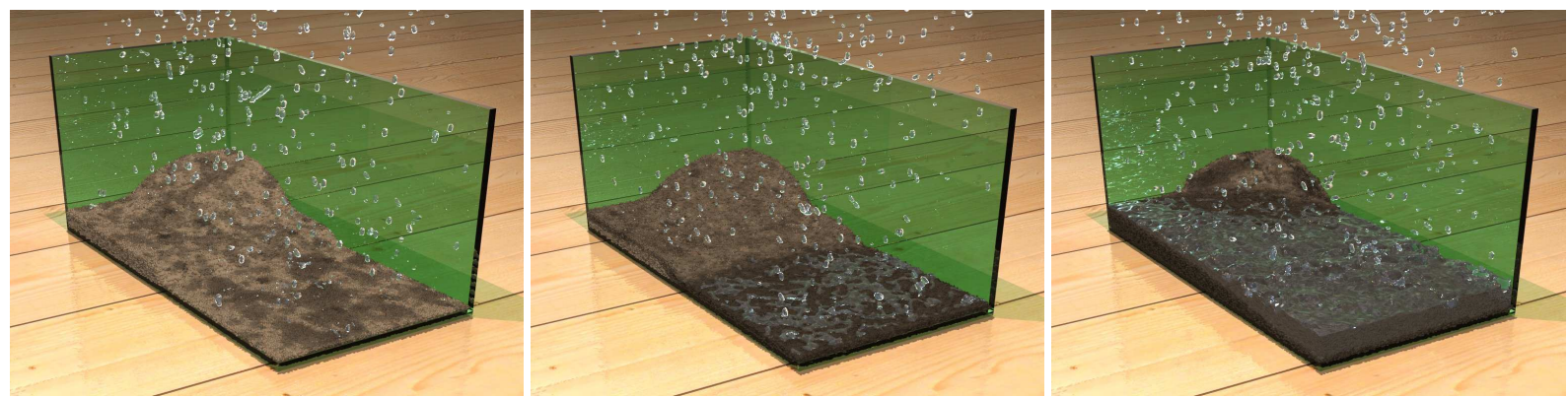

Figure 5: A rain shower on dry soil. As the soil becomes saturated a layer of water forms on top and the soil turns to mud.

Their method achieves interactive rates by implementing the algorithm on the GPU. In principle our fluid and granular material simulation framework could be accelerated using the same GPGPU technique. However, the porous flow framework creates multi-resolution fluid particles which may hinder such a GPGPU implementation (e.g. typically nearest-neighbors are searched on a grid with only a limited amount of particles per cell).

\section{Conclusion}

We have shown how the fluid-sand model of [ZB05] can be simulated in a unified SPH framework. Furthermore, by using porous particles [LAD08] for the sand volume we are able to fully couple fluids and granular materials. Simulating both sand and fluid as continuous volumes enables animations consisting of larger amounts of sand interacting with fluid compared to previous approaches. Our method enables new effects such as fluid percolating through sand, rigid granular structures and dirt turning into mud.

Acknowledgements Toon Lenaerts is funded by a Ph.D. grant of the Institute for the Promotion of Innovation through Science and Technology in Flanders (IWT-Vlaanderen). We would like to thank the reviewers for their contributing remarks. We also thank Stanford University for making available the Bunny model.

\section{References}

[ABC*07] Ammann C., Bloom D., Cohen J. M., Courte J., Flores L., Hasegawa S., Kalaitzidis N., Tornberg T., TREWEEK L., WinTER B., YANG C.: The birth of sandman. In SIGGRAPH 2007 sketches (2007).

[APKG07] Adams B., Pauly M., Keiser R., Guibas L. J.: Adaptively sampled particle fluids. In SIGGRAPH '07: ACM SIGGRAPH 2007 papers (New York, NY, USA, 2007), ACM, p. 48 .

[BT07] BECKER M., TESCHNER M.: Weakly compressible SPH for free surface flows. In SCA '07: Proceedings of the 2007 ACM SIGGRAPH/Eurographics symposium on Computer animation (2007), pp. 209-217.

[BTT09] BecKer M., Tessendorf H., Teschner M.: Direct forcing for lagrangian rigid-fluid coupling. IEEE Transactions on Visualization and Computer Graphics (2009).

[BYM05] Bell N., Yu Y., Mucha P. J.: Particle-based simulation of granular materials. In SCA '05: Proceedings of the 2005 ACM SIGGRAPH/Eurographics symposium on Computer animation (New York, NY, USA, 2005), ACM Press, pp. 77-86.

[CBP05] Clavet S., Beaudoin P., Poulin P.: Particle-based viscoelastic fluid simulation. In SCA '05: Proceedings of the 2005 ACM SIGGRAPH/Eurographics symposium on Computer animation (New York, NY, USA, 2005), ACM Press, pp. 219 228.

[CGFO06] Chentanez N., Goktekin T. G., Feldman B. E., O'BRIEN J. F.: Simultaneous coupling of fluids and deformable bodies. In SCA '06: Proceedings of the 2006 ACM SIGGRAPH/Eurographics symposium on Computer animation 
(Aire-la-Ville, Switzerland, Switzerland, 2006), Eurographics Association, pp. 83-89.

[ClH96] Chanclou B., Luciani A., Habibi A.: Physical models of loose soils dynamically marked by a moving object. In $C A$ '96: Proceedings of the Computer Animation (Washington, DC, USA, 1996), IEEE Computer Society, p. 27.

[CMT04] CARlson M., Mucha P. J., Turk G.: Rigid fluid: animating the interplay between rigid bodies and fluid. $A C M$ Trans. Graph. 23, 3 (2004), 377-384.

[DC96] Desbrun M., CANi M.-P.: Smoothed particles: A new paradigm for animating highly deformable bodies. In Computer Animation and Simulation '96 (1996), pp. 61-76.

[DC99] Desbrun M., CAni M.-P.: Space-Time Adaptive Simulation of Highly Deformable Substances. Tech. Rep. 3829, INRIA, BP 105 - 78153 Le Chesnay Cedex - France, December 1999.

[FF01] Foster N., FEDKIW R.: Practical animation of liquids. In SIGGRAPH '01: Proceedings of the 28th annual conference on Computer graphics and interactive techniques (New York, NY, USA, 2001), ACM, pp. 23-30.

[FG07] FAlapPi S., Gallati M.: SPH simulation of water waves generated by granular landslides. In Proceedings of the 32 nd Congress of IAHR (2007).

[FM96] Foster N., Metaxas D.: Realistic animation of liquids. Graph. Models Image Process. 58, 5 (1996), 471-483.

[FM97] Foster N., Metaxas D.: Controlling fluid animation. In CGI '97: Proceedings of the 1997 Conference on Computer Graphics International (Washington, DC, USA, 1997), IEEE Computer Society, p. 178.

[GSlF05] Guendelman E., Selle A., Losasso F., Fedkiw R.: Coupling water and smoke to thin deformable and rigid shells. In SIGGRAPH '05: ACM SIGGRAPH 2005 Papers (New York, NY, USA, 2005), ACM Press, pp. 973-981.

[KAD*06] KeISER R., AdAms B., Dutré P., Guibas L. J., PAUly M.: Multiresolution Particle-Based Fluids. Tech. rep., ETH Zurich, 2006.

[KAG*05] KEISER R, AdAMS B. GASSER D, BAZZI P. Dutré P., Gross M.: A unified Lagrangian approach to solidfluid animation. In Proceedings of the Eurographics Symposium on Point-Based Graphics (2005), pp. 125-133.

[LAD08] Lenaerts T., AdAms B., Dutré P.: Porous flow in particle-based fluid simulations. In SIGGRAPH 08: ACM SIGGRAPH 2008 Papers (New York, NY, USA, 2008), ACM, pp. 49:1-8.

[LC87] Lorensen W. E., Cline H. E.: Marching cubes: A high resolution $3 \mathrm{~d}$ surface construction algorithm. In SIGGRAPH 87: Proceedings of the 14th annual conference on Computer graphics and interactive techniques (New York, NY, USA, 1987), ACM Press, pp. 163-169.

[LM93] Li X., Moshell J. M.: Modeling soil: Realtime dynamic models for soil slippage and manipulation. In In Computer Graphics Proceedings, Annual Conference Series (1993), pp. 361-368.

[LZLW05] LIU Y., ZHU H., LIU X., WU E.: Real-time simulation of physically based on-surface flow. The Visual Computer (2005).

[MCG03] MÜller M., Charypar D., Gross M.: Particlebased fluid simulation for interactive applications. In $S C A$ '03: Proceedings of the 2003 ACM SIGGRAPH/Eurographics symposium on Computer animation (Aire-la-Ville, Switzerland, Switzerland, 2003), Eurographics Association, pp. 154-159.

[Mon92] Monaghan J.: Smoothed particle hydrodynamics. Annual Revision on Astronomy and Astrophysics 30 (1992), 543574.
[Mon05] Monaghan J. J.: Smoothed particle hydrodynamics. Reports on Progress in Physics 68, 8 (August 2005), 1703-1759.

[MP89] Miller G., Pearce A.: Globular dynamics: A connected particle system for animating viscous fluids. Computers and Graphics 13, 3 (1989), 305-309.

[MSKG05] Müller M., Solenthaler B., Keiser R., GROSS M.: Particle-based fluid-fluid interaction. In SCA '05: Proceedings of the 2005 ACM SIGGRAPH/Eurographics symposium on Computer animation (New York, NY, USA, 2005), ACM Press, pp. 237-244.

[ON03] OnOUe K., Nishita T.: Virtual sandbox. In Proc. of Pacific Conference on Computer Graphics and Applications (2003), p. 253.

[PCGFMD08] Pla-Castells M., García-Fernandez I., MARTINEZ-DURA R. J.: Physically-based interactive sand simulation. In Eurographics 2008 - Short Papers (2008), Mania K. Reinhard E., (Eds.), pp. 21-24.

[PKA*05] Pauly M., Keiser R., Adams B., Dutré P., Gross M., GuiBas L. J.: Meshless animation of fracturing solids. In SIGGRAPH '05: ACM SIGGRAPH 2005 Papers (New York, NY, USA, 2005), ACM Press, pp. 957-964.

[PTB*03] Premoze S., TASdizen T., Bigler J., LeFohn A., WhitAKer R. T.: Particle-based simulation of fluids. Eurographics 2003 / Computer Graphics Forum 22, 3 (2003), 401410 .

[RSKN08] RUNGJIRATANANON W., SZEGo Z., KANAMORI Y., NishitA T.: Real-time animation of sand-water interaction. In Computer Graphics Forum (Pacific Graphics 2008) (2008), vol. 27, pp. 1887-1893.

[SOH99] Sumner R., O'BRIEN J. F., Hodgins J. K.: Animating sand, mud, and snow. Computer Graphics Forum 18, 1 (March 1999), 17-26.

[SSP07] Solenthaler B., SChläfli J., PAJAROla R.: A unified particle model for fluid-solid interactions. Computer Animation and Virtual Worlds 18, 1 (2007), 69-82.

[Sta99] STAM J.: Stable fluids. In SIGGRAPH '99: Proceedings of the 26th annual conference on Computer graphics and interactive techniques (New York, NY, USA, 1999), ACM Press/Addison-Wesley Publishing Co., pp. 121-128.

[WCMT07] Wojtan C., Carlson M., Mucha P. J., Turk G.: Animating corrosion and erosion. In Eurographics Workshop on Natural Phenomena (2007), pp. 15-22.

[ZB05] ZHU Y., BRIDSON R.: Animating sand as a fluid. In SIGGRAPH '05: ACM SIGGRAPH 2005 Papers (New York, NY, USA, 2005), ACM Press, pp. 965-972. 